\title{
Parieto-motor Cortical Dysfunction in Primary Cervical Dystonia
}

\author{
Paolo Porcacchia a, Francisco J. Palomar ${ }^{\mathrm{a}, \mathrm{b}}$, María T. Cáceres-Redondo ${ }^{\mathrm{a}}$, \\ Ismael Huertas-Fernández a , Juan F. Martín-Rodríguez ${ }^{\mathrm{a}}$, Fátima Carrillo a \\ Giacomo Koch ${ }^{\mathrm{c}, \mathrm{d}}$, Pablo Mir ${ }^{\mathrm{a}, \mathrm{b}, *}$

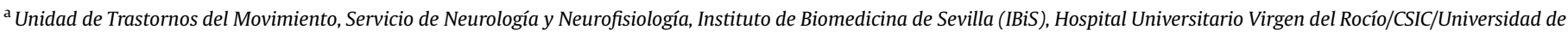 \\ Sevilla, Seville, Spain \\ ${ }^{\mathrm{b}}$ Centro de Investigación Biomédica en Red sobre Enfermedades Neurodegenerativas (CIBERNED), Spain \\ ' Stroke Unit, Dipartimento di Neuroscienze, Università di Roma Tor Vergata, Rome, Italy \\ ${ }^{\mathrm{d}}$ Laboratorio di Neurologia Clinica e Comportamentale, Fondazione S. Lucia I.R.C.C.S., Rome, Italy
}

\section{A R T I C L E I N F O}

Article history:

Received 28 January 2014

Received in revised form

17 June 2014

Accepted 17 June 2014

Available online 17 July 2014

\section{Keywords:}

Dystonia

Hypokinesia

Parietal lobe

Transcranial magnetic stimulation

\begin{abstract}
A B S T R A C T
Background: Dystonia is considered as a motor network disorder involving the dysfunction of the posterior parietal cortex, a region involved in preparing and executing reaching movements.

Objective/hypothesis: We used transcranial magnetic stimulation to test the hypothesis that cervical dystonic patients may have a disrupted parieto-motor connectivity.

Methods: We enrolled 14 patients with primary cervical dystonia and 14 controls. A paired-pulse transcranial magnetic stimulation protocol was applied over the right posterior parietal cortex and the right primary motor area. Changes in the amplitudes of motor evoked potential were analyzed as an index of parieto-motor effective connectivity. Patients and healthy subjects were also evaluated with a reaching task. Reaction and movement times were measured.

Results: In healthy subjects, but not in dystonic patients, there was a facilitation of motor evoked potential amplitudes when the conditioning parietal stimulus preceded the test stimulus applied over the primary motor area by $4 \mathrm{~ms}$. Reaction and movement times were significantly slower in patients than in controls. In dystonic patients, the relative strength of parieto-motor connectivity correlated with movement times. Conclusions: Parieto-motor cortical connectivity is impaired in cervical dystonic patients. This neurophysiological trait is associated with slower reaching movements.
\end{abstract}

(c) 2014 Elsevier Inc. All rights reserved.

\section{Introduction}

Dystonia is a movement disorder characterized by excessive involuntary muscle contraction. Primary focal dystonias are more common than primary generalized dystonias [1]. Cervical dystonia is the most common form of focal dystonia [2].

The pathophysiology of dystonia is not completely understood. Impaired inhibition at multiple levels of the central nervous system

Funding: This work was supported by grants from the Ministerio de Economía y Competitividad de España (SAF2007-60700), the Instituto de Salud Carlos III (CP08/ 00174, PI10/01674, PI13/01461), the Consejería de Economía, Innovación, Ciencia y Empresa de la Junta de Andalucía (CVI-02526, CTS-7685), the Consejería de Salud y Bienestar Social de la Junta de Andalucía (PI-0377/2007, PI-0741/2010, PI-04372012, PI-0471/2013), the Sociedad Andaluza de Neurología, the Fundación Alicia Koplowitz, the Fundación Mutua Madrileña and the Jaques and Gloria Gossweiler Foundation.

The authors report no conflict of interest.

* Corresponding author. Unidad de Trastornos del Movimiento, Servicio de Neurología y Neurofisiología Clínica, Hospital Universitario Virgen del Rocío. Av. Manuel Siurot s/n., 41013 Sevilla, Spain. Tel.: +34 955012593; fax: +34 955012597.

E-mail address: pmir@us.es (P. Mir). is present [3], with alterations of motor circuits involving the basal ganglia [4], the cerebellum [5,6] and the sensorimotor cortex [7,8]. Recent evidences seem to suggest that the dysfunction of the motor network involves other cortical areas such as the parietal cortex $[9,10]$. Neuropathological and neuroimaging evidences reviewed in a recent paper [11], suggest that the parietal region is implicated in different forms of dystonia, in terms of changes of regional blood flow or gray matter volume. A reduction of the parietal cortex activation was detected during imaging of movement in patients with cervical dystonia [12]. Moreover, after repetitive transcranial magnetic stimulation (TMS) over the parietal cortex, the activation of the parietal cortex during motor execution, measured by functional magnetic resonance imaging (fMRI), was reduced in patients with cervical dystonia [13].

In the current study we aim to explore, with a TMS technique, the connectivity among the posterior parietal cortex (PPC) and the ipsilateral primary motor area (M1) [14] in cervical dystonia. With this method a conditioning stimulus (CS) is first used to activate putative pathways, while a second test stimulus (TS), delivered over M1 a few milliseconds later, is used to explore changes in 
excitability produced by the input $[14,15]$. In healthy subjects, a conditioning TMS pulse applied over the right PPC is able to increase the excitability of the hand area of the right M1 [16].

The PPC-M1 interaction is crucial in preparation and planning of reaching and grasping movements toward visual targets [17-19], as well as in visuospatial mechanisms that affect temporal performance, accuracy and variability [18,20]. Reaching movements have been proved as a reliable behavioral correlate of the PPC-M1 interaction, because the excitability of this pathway varies during the task [17]. Dystonic patients may show behavioral motor task abnormalities; in fact reaction time task studies in patients with idiopathic torsion dystonia showed that initiation and execution responses were slower than in control subjects [21].

Hence our aim was to study PPC-M1 connectivity in cervical dystonic patients, at rest, using this paired-pulse TMS protocol. Moreover we hypothesize that the efficacy of PPC-M1 interaction could be directly related to the slowness in movement time that characterizes cervical dystonic patients.

\section{Methods and materials}

Subjects

Fourteen right-handed patients ( 5 men, 9 women, mean age $48 \pm 14$ years, disease duration $8 \pm 5$ years) affected by primary cervical dystonia (Table 1) were recruited from the Movement Disorders Outpatient Clinic at the Hospital Universitario Virgen del Rocío in Seville, Spain. Diagnosis of cervical dystonia was made by expert neurologists, based on clinical and anamnestic findings. The assessment included a complete Toronto Western Spasmodic Torticollis Rating Scale (TWSTRS) and the Burke-Fahn-Marsden Dystonia Rating Scale (BFMDRS). The TMS experiments were performed at least 3 months after the last botulinum toxin injection. All other oral drugs were stopped $48 \mathrm{~h}$ before the TMS experiments.

Fourteen age-matched ( 6 men and 8 women, $48 \pm 15$ years), healthy, right-handed volunteers served as control subjects. They were recruited from the hospital and research staff. The study was approved by the local ethics committee and all the subjects gave written informed consent.

\section{Experimental procedure}

\section{PPC-M1 connectivity}

Subjects were seated comfortably and we followed the same design, electromyography (EMG) recordings and off-line peak-to- peak amplitude analysis that were used in a previous study [16,22]. The paired-pulse stimulation technique was used with two different high-power Magstim $200^{2}$ machines (Magstim Co., Whitland, Dyfed, UK). The hand motor area of the right M1 was found at the point where the largest motor evoked potential (MEP) from the contralateral FDI muscle was elicited and the optimal position was marked on the scalp, to ensure the minimum displacement during the experiment. The intensity of the TS was adjusted to elicit $1 \mathrm{mV}$ MEP amplitude in the relaxed FDI. The test stimulator was connected to a figure-of-eight coil with a $55 \mathrm{~mm}$ external diameter. The coil was positioned at a $45^{\circ}$ angle from the midline to induce a posterior-anterior current flow. The conditioning stimulator was connected to a standard figure-of-eight shaped coil with a $70 \mathrm{~mm}$ external diameter, positioned over the P4 position (10-20 EEG system) (Fig. 1A). This site is situated in the inferior parietal lobule [18,23-26], that is part of the posterior parietal cortex, and it is defined by the following Tailarach coordinates: $38.4 \pm 6.1$, $-67.2 \pm 4.4$, and $46.3 \pm 5.8 \mathrm{~mm}$ [27]. The center of the coil was positioned over P4 tangentially to the skull and with the handle pointing downward and slightly medial $\left(10^{\circ}\right)$. MRI-guided frameless stereotaxy (Brainsight Frameless; Rogue Research, Montreal, Quebec, Canada) was used in all subjects to ensure the minimum displacement during PPC stimulation (Fig. 1B). We performed three blocks with different intensities of the CS set at 70\%, 90\% and 110\% of the resting motor threshold (RMT). RMT was tested according to international standards [28], with the figure-of-eight shaped coil (70 mm diameter). Inter-stimulus intervals (ISI) between CS and TS were 2, 4, 6, 8, 10, 15, and $20 \mathrm{~ms}$ (Fig. 1A). Each block consisted of 20 trials only with TS and 10 trials with CS + TS for each ISI (total 90 trials per block). In each block the trials were randomly intermingled with an inter-trial time of $5 \mathrm{~s}$. The order of presentation of the blocks of trials varied randomly in control and patients.

\section{Reaction time task}

We used a choice reaction time task similar to that adopted previously [17,22]. All subjects sat comfortably in a 45-cm-high straight-back chair facing a table, $120 \mathrm{~cm}$ wide and $60 \mathrm{~cm}$ deep. On the opposite edge of the table an upright, home-made flat wooden panel was fixed, $80 \mathrm{~cm}$ wide and $50 \mathrm{~cm}$ high, placed at $60 \mathrm{~cm}$ distance from the subject. Subjects placed the index finger of their left hand on an upraised bump (2.5 cm-diameter coin), that acted as starting point, on the table surface. Peripheral targets comprised $2 \mathrm{~cm}$ diameter upraised bumps, positioned $20 \mathrm{~cm}$ left or right of a fixation cross at a viewing distance of $60 \mathrm{~cm}$ (Fig. 1C). The starting point

Table 1

Clinical characteristics of patients with primary cervical dystonia.

\begin{tabular}{|c|c|c|c|c|c|c|c|}
\hline No. & Sex & Age (years) & $\begin{array}{l}\text { Disease duration } \\
\text { (years) }\end{array}$ & TWSTRS & BFMDRS & $\begin{array}{l}\text { Dominant hand/ } \\
\text { head deviation }\end{array}$ & Treatment (mg/day) \\
\hline 1 & $\mathrm{M}$ & 39 & 3 & 26.7 & 13.5 & $\mathrm{R} / \mathrm{L}$ & BT \\
\hline 2 & $\mathrm{~F}$ & 34 & 15 & 16 & 12 & $\mathrm{R} / \mathrm{R}$ & BT \\
\hline 3 & M & 34 & 9 & 40.75 & 17.5 & $\mathrm{R} / \mathrm{R}$ & BT \\
\hline 4 & M & 32 & 15 & 32.25 & 8 & $\mathrm{R} / \mathrm{R}$ & BT \\
\hline 5 & M & 32 & 5 & 55.75 & 22 & $\mathrm{R} / \mathrm{R}$ & BT, clonazepam (2) \\
\hline 6 & $\mathrm{~F}$ & 44 & 3 & 44 & 25 & $\mathrm{R} / \mathrm{L}$ & BT, clonazepam (10) \\
\hline 7 & $\mathrm{~F}$ & 48 & 8 & 22.5 & 7.5 & $\mathrm{R} / \mathrm{L}$ & BT \\
\hline 8 & $\mathrm{~F}$ & 68 & 10 & 32 & 6 & $\mathrm{R} / \mathrm{L}$ & BT \\
\hline 9 & M & 64 & 16 & 36 & 26 & $R / R$ & BT, trihexyphenidyl (6) \\
\hline 10 & $\mathrm{~F}$ & 66 & 9 & 9.75 & 21 & $\mathrm{R} / \mathrm{L}$ & Trihexyphenidyl (6) \\
\hline 11 & $\mathrm{~F}$ & 55 & 2 & 12 & 1.5 & $\mathrm{R} / \mathrm{R}$ & BT \\
\hline 12 & $\mathrm{~F}$ & 68 & 4 & 30.75 & 23.5 & $\mathrm{R} / \mathrm{L}$ & BT \\
\hline 13 & $\mathrm{~F}$ & 40 & 2 & 31 & 38.2 & $R / R$ & BT \\
\hline 14 & $\mathrm{~F}$ & 48 & 8 & 25 & 39.5 & $\mathrm{R} / \mathrm{L}$ & BT \\
\hline
\end{tabular}

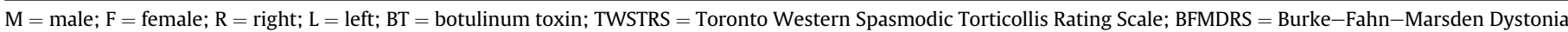
Rating Scale. 

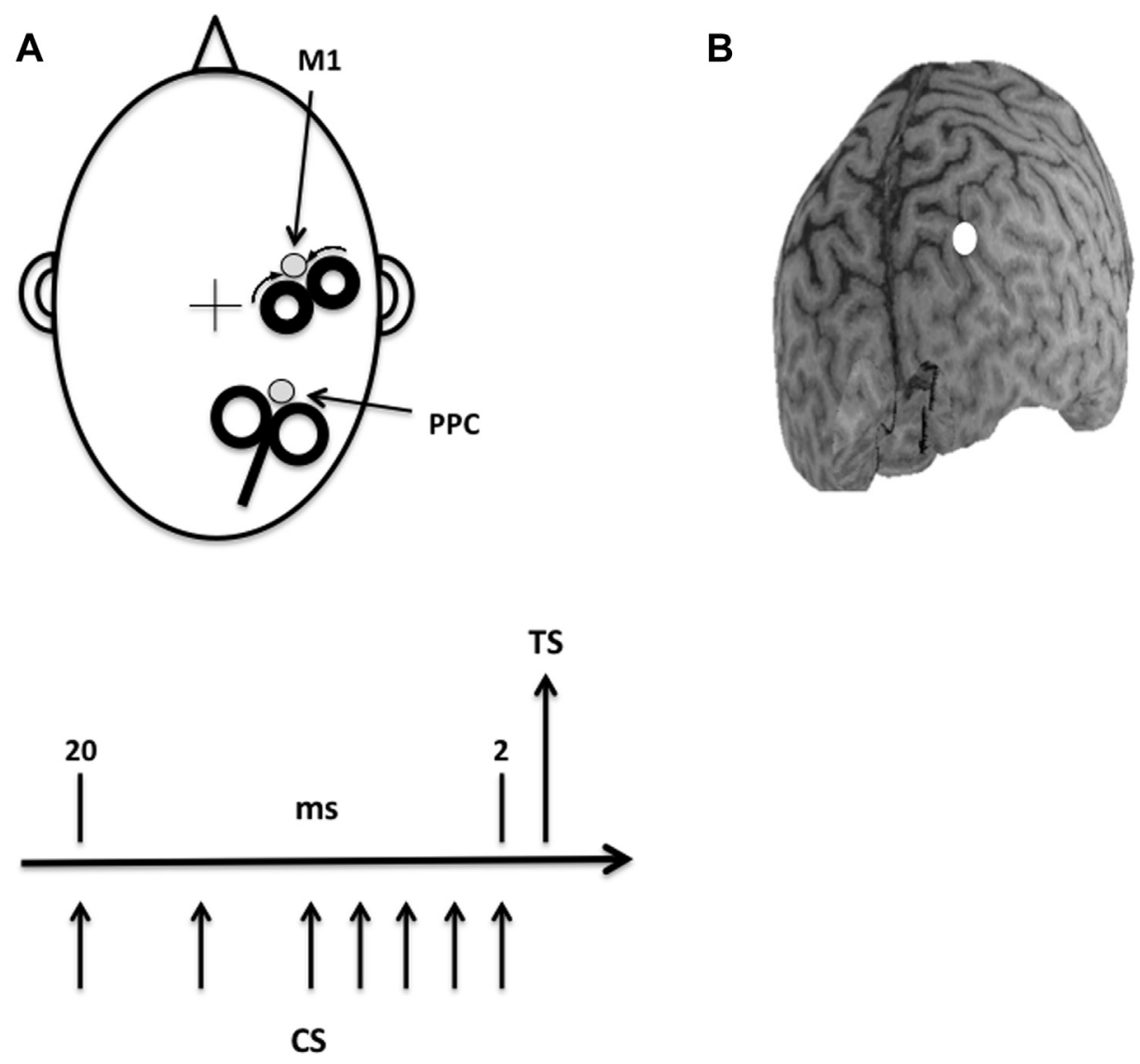

C
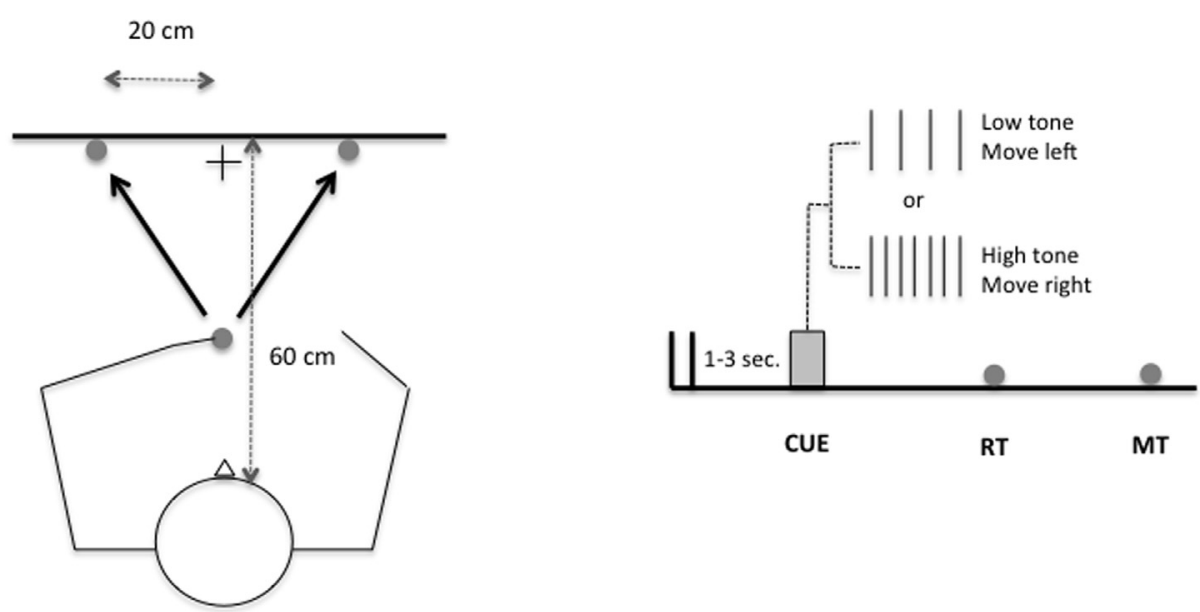

Figure 1. (A) TMS procedure. Shorter arrows represent the posterior parietal cortex (PPC) conditioning stimulus (CS), that preceded test stimulus (TS) (longer arrow). (B) PPC stimulation using neuronavigation system. (C) Reaction task. After the imperative sound, the subjects reached to the left or right target. Modified from Ref. [22].

and both peripheral targets were worn using three independent proximity sensors of plastic optic fiber (reflective fibre optic sensor, LL3-DT01, SICKOPTEX, Japan). This kind of plastic optic fiber sensor produces a positive square signal during sensor activation (sensor switch on) or a negative square signal during sensor deactivation (sensor switch off). Sensor responses were digitally converted by a Power1401 (Cambridge Electronic Devices, UK) and recorded with SIGNAL software (Cambridge Electronic Devices, UK). Each trial began with an auditory warning followed by the imperative auditory signal randomly given $1-3 \mathrm{~s}$ later. Subjects were required to reach toward and touch the peripheral left or right target as soon as they heard the imperative sound. The imperative signal consisted of either a high $(800 \mathrm{~Hz}, 30 \mathrm{~ms})$ or low $(200 \mathrm{~Hz}, 30 \mathrm{~ms})$ frequency tone pulse that indicated which peripheral target subjects had to reach (high meaning reach right, low meaning reach left, or vice versa). All subjects performed a block of 80 trials. A training block of 35 trials was performed before starting with the 80 trial block. The inter-trial interval was $6 \mathrm{~s}$. At the start of each block, the high and low tones were assigned randomly to indicate the side target to reach (left or right). These instructions were counterbalanced within and across 
subjects. In consequence, each trial had a single reaction time (RT) that was defined as the time between the imperative sound and the switching off of the starting point sensor, corresponding to the first movement of the left index finger. Movement time (MT) was defined as the time between the RT and the switching on of the corresponding reached peripheral target sensor. TMS experiment and reaction time task were performed in two separate days at least one week apart. All the control subjects and twelve of the fourteen cervical dystonic patients performed the reaction time task. Two patients did not attend to the reaction time task session and they were lost on follow-up.

\section{Data analysis}

Shapiro-Wilk test was used to check the normal distribution of the data. Parametric or non-parametric tests were used for data with or without normal distribution respectively. Magnetic stimulation intensities, clinical and demographic data were analyzed using Wilcoxon and Mann-Whitney $U$ tests, depending on data type. In PPC-M1 connectivity experiments, PPC-conditioned MEP amplitudes were normalized to non-conditioned one (TS alone). Normalized data were analyzed using repeated measures ANOVA, with PPC CS "INTENSITY" (70\%, 90\% and 110\% of RMT) and "ISI" (2, 4, $6,8,10,15$, and $20 \mathrm{~ms}$ ) as the within-subject factors and "GROUP" (patients vs. control) as the between-subject factor. A significant interaction in the ANOVA was followed by post-hoc paired $t$-test analysis with Bonferroni correction. The Greenhouse-Geisser correction was used for non spherical data and Mauchly's test examined for sphericity.
In the reaction time experiment, RT and MT were calculated separately for the left and right target. Independent and repeated measures t-tests were used to analyze RT and MT data. Pearson's and Spearman's correlations between conditioned MEP amplitudes at $4 \mathrm{~ms}$ ISI (CS 90\% of RMT), RT and MT, TWSTRS and BFMDRS scores head deviation side and age was performed to explore the clinical to functional relationship. A $P$ value of $<0.05$ was considered to be statistically significant in all analyses. All statistical analyses were carried out using IBM SPSS Statistics 20 software.

\section{Results}

\section{Demographic data and magnetic stimulation intensities}

No significant differences were found in demographic data between patients and control subjects. RMT and TS intensities were not different among groups ( $P=0.92$ and $P=0.98$ respectively). Mean RMT values were $41.2 \% \pm 6.4$ of maximum stimulator output in control subjects and $41.0 \% \pm 6.9$ in patients. Mean TS intensity values were $51.2 \% \pm 8.6$ of maximum stimulator output in control subjects and $51.1 \% \pm 7.8$ in patients.

\section{PPC-M1 connectivity}

Factorial repeated measures ANOVA showed a significant GROUP $\times$ ISI $(F=2.890 ; P=0.011)$ and GROUP $\times$ ISI $\times$ INTENSITY $(F=2.184 ; P=0.012)$ interactions. Paired $t$-test analyses revealed a significant difference between control and dystonic groups at $4 \mathrm{~ms}$ ISI for a CS intensity of 90\% RMT $(P<0.001)$ (Fig. 2A and B). Controls showed an effect of double pulse intervention $(P=0.011$ at $4 \mathrm{~ms})$

A

PPC-M1 90\%

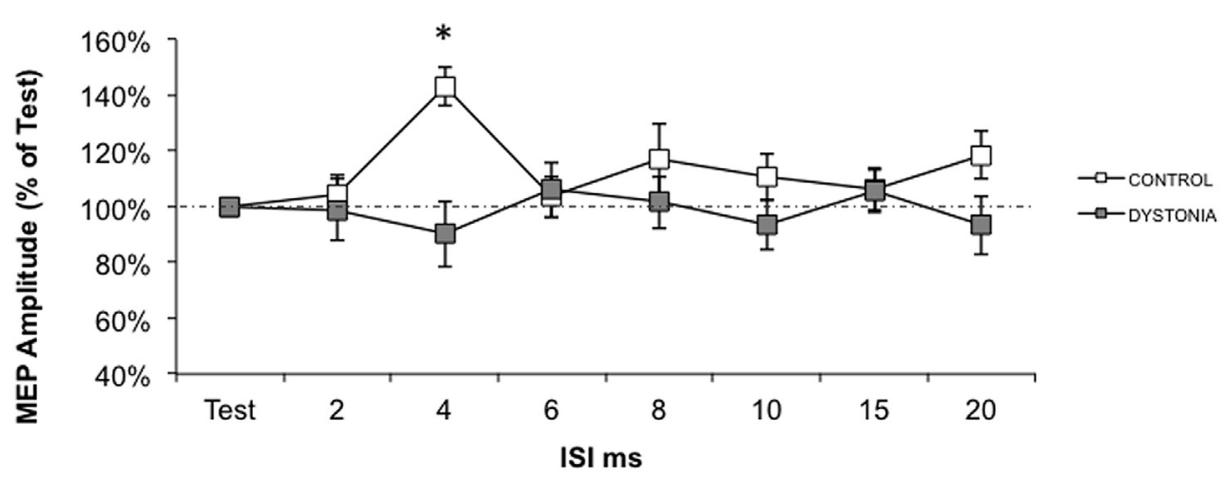

B

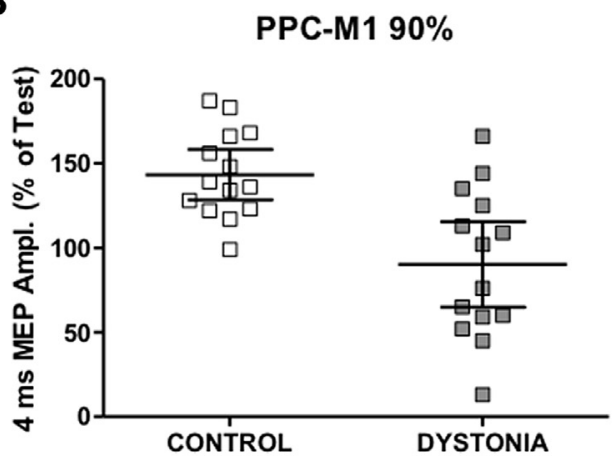

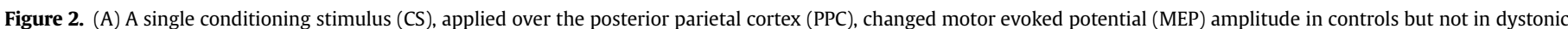

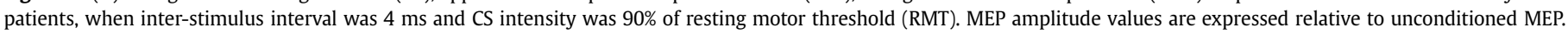

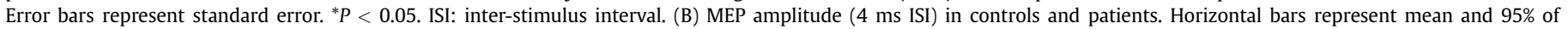
confidence interval. 
while no effect was present in patients, showing that there was an MEP facilitation when the PPC CS preceded the TS over M1 by $4 \mathrm{~ms}$ at a CS intensity of $90 \%$ of the RMT in control subjects and that this effect was not observed in the group of dystonic patients. No differences were observed between control and dystonic patients groups at any other ISI or CS intensity (Supplementary Fig. 1).

\section{Reaction time task}

RT as well as MT both toward the left and right sides were significantly lower in control subjects than in patients $\left(P_{\mathrm{S}}\right.$ less than 0.007 , Figs. 3 and 4 ). When tested for right-left difference, both groups showed minor left movement time values respect to the right ones $(P<0.0001$ in patients and $P=0.002$ in controls) without differences in reaction time values. No correlation was found between RT and MT in both groups.
We then correlated the individual values obtained in the TMS experiments for the PPC-M1 connectivity (with the intensity of CS at 90\% RMT, 4 ms ISI) with the individual RTs and MTs. In control subjects MEP amplitude did not correlate with either RT or MT. However, in cervical dystonic patients MEP amplitude significantly correlated with MT toward both the left side $(P=0.016, r=-0.674)$ and the right side $(P=0.032, r=-0.619)$ but not with RT (Figs. 3 and 4 ).

No differences between the two groups were found in decision errors. There were no anticipation errors (RT $<150 \mathrm{~ms}$ ), no omission errors (the subject do not move after the imperative sound) and no abnormal long responses (RT $+\mathrm{MT}>2 \mathrm{~s}$ ).

In patient group, no significant correlation was found between TWSTRS, BFMDRS scores, head deviation side, and age with MEP amplitude (CS at 90\% RMT, 4 ms ISI) or with RT and MT values.

A

LEFTWARD MOVEMENTS

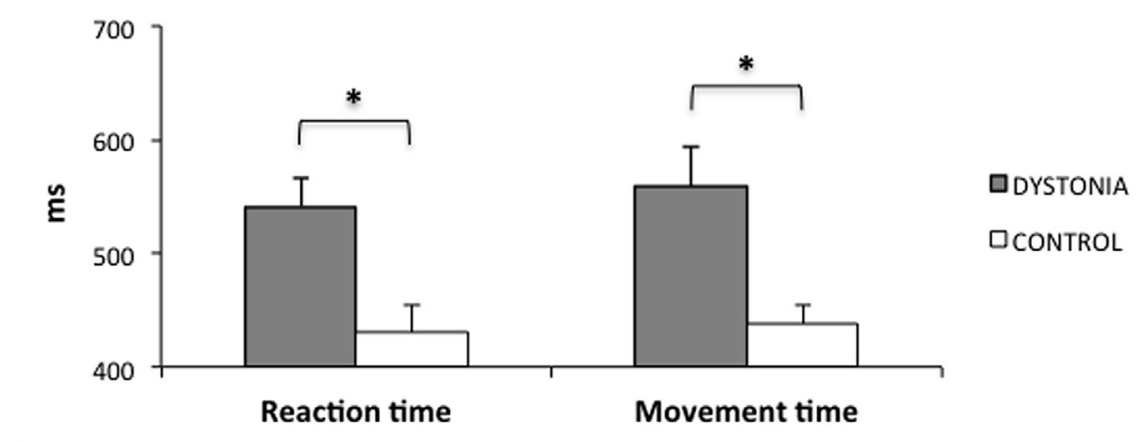

B

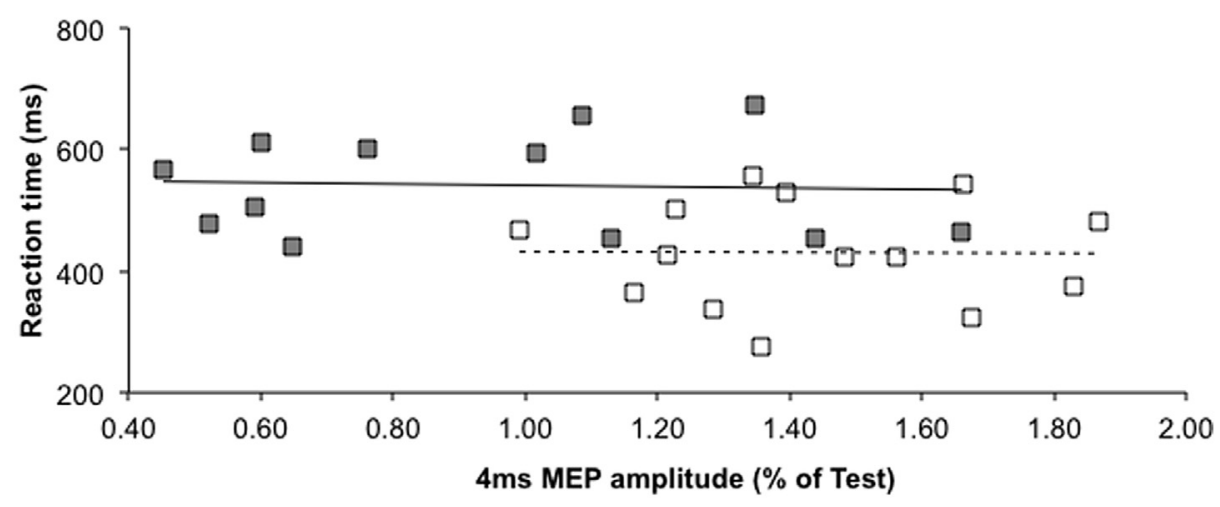

C

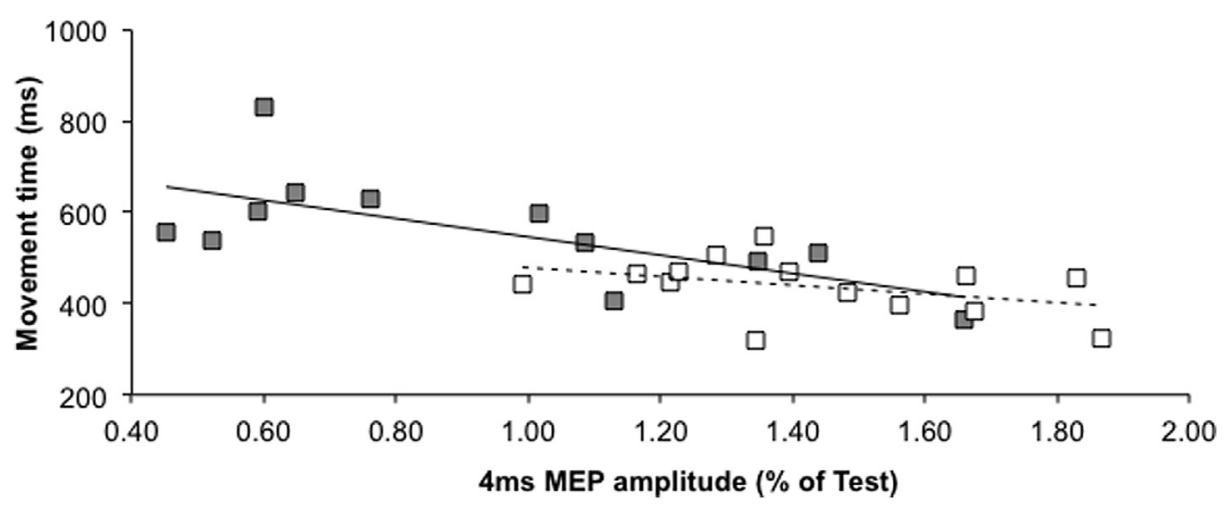

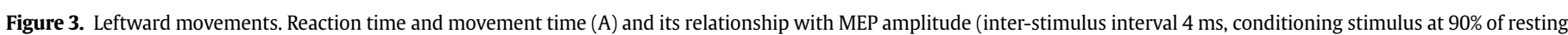
motor threshold) (B, C). A significant correlation was observed only in patients, between MEPs and movement times $(P=0.016, r=-0.674$; continuous line $(C)$ ). 
A RIGHTWARD MOVEMENTS

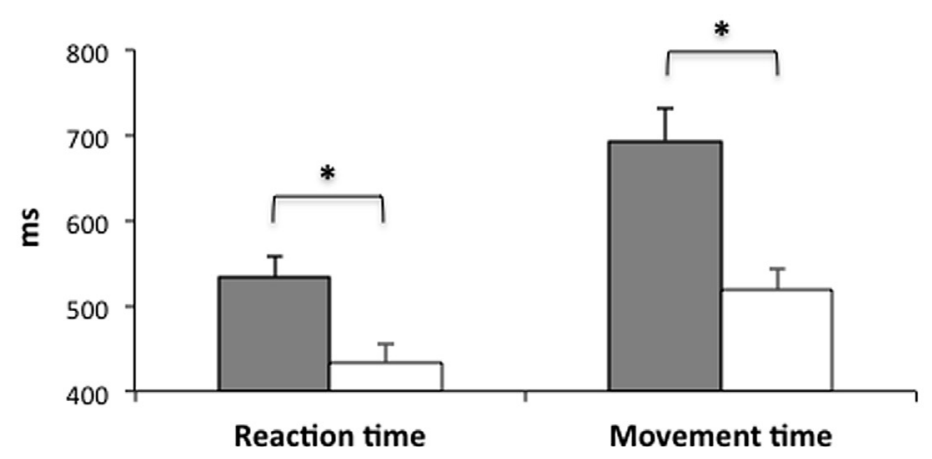

口DYSTONIA

口CONTROL

B

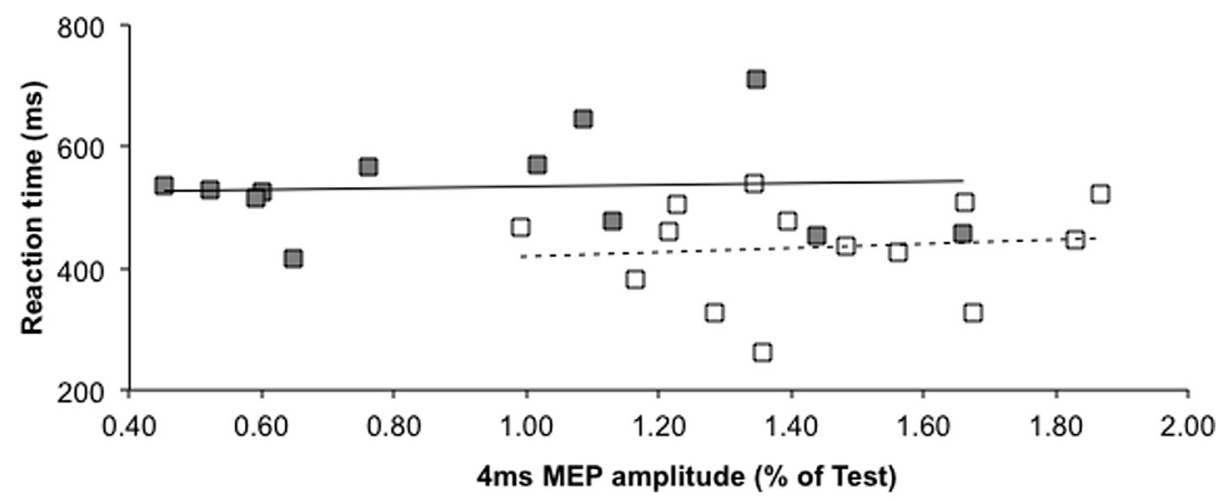

C

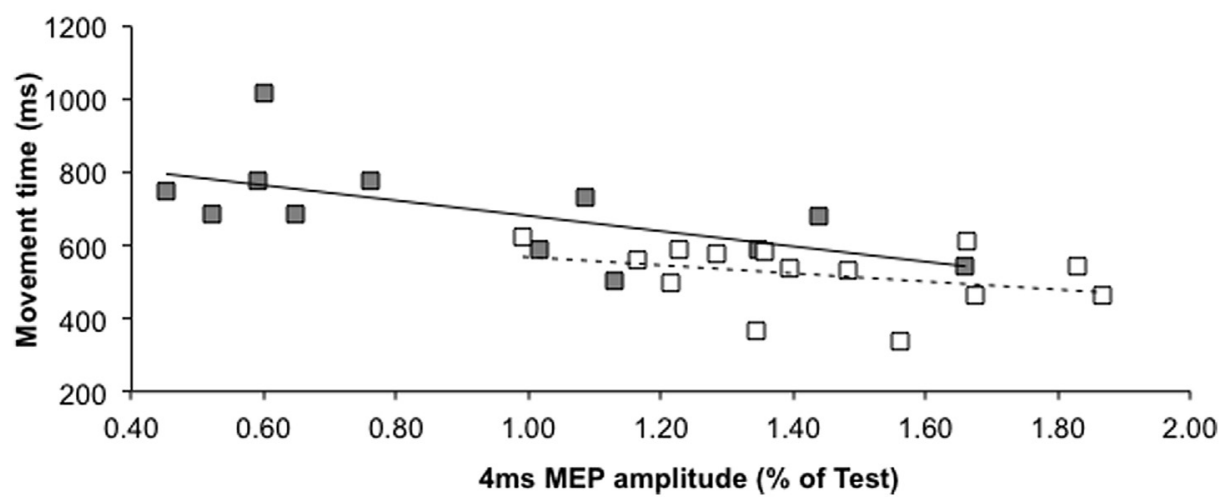

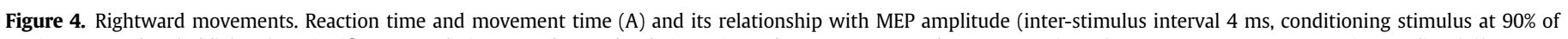
resting motor threshold) (B, C). A significant correlation was observed only in patients, between MEPs and movement times $(P=0.032, r=-0.619$; continuous line $(C)$ ).

\section{Discussion}

This study shows that parieto-motor cortical connectivity is impaired in cervical dystonic patients at rest and that parietal dysfunction correlates with slower movement time in a choice reaction task.

Exploring parieto-motor cortical connectivity with TMS is a well-established paradigm both in healthy subjects [16-18] and in neurological patients $[22,29,30]$. The activation of cortico-cortical projections arising from the inferior parietal lobe and/or intraparietal sulcus and terminating in M1 is probably responsible for the early peak (4-8 ms) that is observed with this protocol [16]. The transfer of this information probably occurs through fibers of the superior longitudinal fasciculus [31], either through direct projection or an indirect pathway involving the ipsilateral ventral premotor cortex [18]. In our study, when CS over PPC was set at $90 \%$ of
RMT and preceding TS by $4 \mathrm{~ms}$, MEPs arising from M1 were increased in control subjects but not in patients, suggesting the existence of a dysfunction of parieto-frontal cortico-cortical connectivity in cervical dystonic patients.

Parieto-motor impairment cannot be easily ascribed to a different threshold of activation of the cortico-cortical output originating from PPC (both CS at 70\% and $110 \%$ of RMT failed to modulate M1 response) but probably reflects some intrinsic changes or some loss of functionality of the PPC neuronal population and/or abnormal influences of PPC on ipsilateral M1 circuits. A previous study using fMRI showed decreased parietal activation during movement in cervical dystonic patients [13], and task-independent (at rest) alterations of premotor-parietal circuits in writer's cramp patients [32]. Our study follows this precedent, revealing, with a TMS paradigm, an at rest impairment of the parieto-motor circuit in cervical dystonic patients. It can be argued that the same M1 
circuits can be implicated in this altered response, and the present study did not test it specifically. Anyway M1 excitability should not grossly differ between patients and controls given that RMT and the intensity needed for test stimuli are very similar in the two groups.

Slower initiation and execution responses were observed in patients with idiopathic torsion dystonia [21] and, similarly, voluntary head movements have been reported to be slow in cervical dystonic patients [33-36]. Interestingly, a recent study in cervical dystonia proved an impairment of the coordination of large gaze reorientations as well as a reduction of body segmental velocity (in particular, trunk bradykinesia), leading to gross prolongations in target acquisition time [37], and supporting the existence of "bradykinesia" in cervical dystonic patients. As expected, in our group of cervical dystonic patients we found slower reaction and movement times. We also found a negative relationship between MEP amplitude (at 4 ms ISI, with PPC-CS at 90\% of RMT) and movement time, pointing toward the role of parietal dysfunction in movements' slowness. Anyway, the design of our study does not permit to establish a causal relationship between the parietal impairment and movements' slowness in cervical dystonic patients. Future studies, applying TMS during the reaction time task or manipulating PPC function by inhibitory repetitive TMS before the motor task, could clarify the effective causal relationship between PPC function and "bradykinesia." The role of this parieto-motor functional connection in the pathophysiology of bradykinesia has been recently suggested in Parkinson's disease, with the same experimental procedure [22]. This analogy should be carefully evaluated for at least two reasons. First the nature of bradykinesia may be different in the two conditions. In cervical dystonic patients, the close association between head-on-trunk velocity and trunk velocity [37] supports the view that the "bradykinesia" is at least in part "secondary" to the slow head movement, as opposed to "primary" bradykinesia in Parkinsonism. Second, this analogy might suggest that impaired PPC-M1 connectivity and its relation to reaction time is an unspecific trait. If it is present in different type of movement disorders, it may represent an epiphenomenon of an abnormal output of other nervous structures and one can hypothesize that basal ganglia could be implicated. Further studies are needed to prove if these results can be extended to other dystonia types or other movement disorders.

In our patients we cannot exclude impairment in the programming (if left or right) and planning of reaching movements, the ability to initiate movement promptly and visual motor-integration during movement. Parieto-motor dysfunction may affect all these different components $[19,20]$, leading to a progressive summation of time delays, beginning from the first proposal of the movement and persisting until the end of the reaching task. Indeed our cervical dystonic patients showed not only prolonged MT but also slower RTs, while, in Parkinson's disease patients, RTs were not slower [22]. In addition, motor cortical excitability that precedes a voluntary movement is abnormally modulated in patients with upper limb dystonia [38]. They cannot properly recruit the neurons or circuits required to perform the movement and this could also contribute in making reaction time slower.

When analyzing right-left differences, we observed that leftside MT values were smaller than right side ones in both groups. The explication can be found in the experiment setting, for the reason that, when reaching the right target, the left arm undergoes a bigger displacement than toward the left target. The fact that longer right side MTs were observed it in both control and dystonic group support this hypothesis.

Interestingly we found a relation of parieto-motor activity with MT toward both the ipsilateral and the contralateral space, while a functional interplay was demonstrated in healthy subjects only toward contralateral directions [17]. Parietal dysfunction could be bilateral and comparable in the two hemispheres and it could be associated to a bilateral increase of MT or, as an unspecific and non causal trait, other mechanisms and structures are implicated, as mentioned before.

The lack of correlation of these parameters with TWSTRS and BFMDRS may indicate a reduced sensibility of clinical scales in detecting subtle neurophysiological and motor task changes in our group of patients or that parieto-motor dysfunction is an adaptive phenomenon, not necessarily correlated with clinical scale.

We chose to explore right PPC-M1 connectivity because, even if parieto-motor facilitation is present bilaterally, in the left hemisphere the time course is quite different [16] and the effects are somewhat milder in term of relative facilitation [17].

Finally, both in controls and in patients, we did not observe a late (15 ms) parieto-motor interaction [16,18,39]. As observed in a previous study [22], a decreased function of non-primary motor areas activity in older subjects [40] may explain these differences.

A limitation of the study is how we set parietal spot. As in a previous study [22], we used de 10-20 electrode system to place the TMS coil and then with the Brainsight we ensured the minimum displacement during the study. So it is possible that we did not localize the optimal facilitatory spot in all subjects. Anyway we used the same procedure in controls and patients and the differences we found between these two groups cannot be ascribed to a better parietal spot location in one group respect to the other.

In conclusion we proved that cervical dystonic patients show a parieto-motor cortical dysfunction that is evident at rest as a task-independent neurophysiological abnormality. The slower movement time during a choice reaction time task could be an epiphenomenon of this parieto-motor impairment. The study supports the hypothesis that parietal cortex is one of the structures involved in the pathophysiology of dystonia, as a network disorder that involves different brain regions [41]. A network in which it may be difficult to distinguish alterations that are causative, adaptive or maladaptive. Finally identifying a neurophysiological-behavioral relationship may permit, in the future, to improve clinical symptoms by producing plastic changes in the corresponding area of the brain.

\section{Acknowledgments}

We would like to thank Juan Manuel Praena Fernández for his help with the statistical analysis, Félix Jesús Pérez Simón for his technical support in the reaction time task and all the patients for their kind participation in this study.

\section{Supplementary data}

Supplementary data related to this article can be found at http:// dx.doi.org/10.1016/j.brs.2014.06.007.

\section{References}

[1] Greene P, Kang UJ, Fahn S. Spread of symptoms in idiopathic torsion dystonia. Mov Disord 1995;10:143-52.

[2] Tarsy D, Simon DK. Dystonia. N Engl J Med 2006;355:818-29.

[3] Hallett M. The neurophysiology of dystonia. Arch Neurol 1998;55:601-3.

[4] Gernert M, Bennay M, Fedrowitz M, Rehders JH, Richter A. Altered discharge pattern of basal ganglia output neurons in an animal model of idiopathic dystonia. J Neurosci 2002;22:7244-53.

[5] Jinnah HA, Hess EJ. A new twist on the anatomy of dystonia: the basal ganglia and the cerebellum. Neurology 2006;67:1740-1.

[6] Teo JT, Van De Warrenburg BP, Schneider SA, Rothwell JC, Bhatia KP. Neurophysiological evidence for cerebellar dysfunction in primary focal dystonia. J Neurol Neurosurg Psychiatry 2009;80:80-3.

[7] Abbruzzese G, Marchese R, Buccolieri A, Gasparetto B, Trompetto C. Abnormalities of sensorimotor integration in focal dystonia: a transcranial magnetic stimulation study. Brain 2001;124:537-45. 
[8] Bäumer T, Demiralay C, Hidding U, et al. Abnormal plasticity of the sensorimotor cortex to slow repetitive transcranial magnetic stimulation in patients with writer's cramp. Mov Disord 2007;22:81-90.

[9] Egger K, Mueller J, Schocke M, et al. Voxel based morphometry reveals specific grey matter changes in primary dystonia. Mov Disord 2007;22:1538-42.

[10] Garraux G, Bauer A, Hanakawa T, et al. Changes in brain anatomy in focal hand dystonia. Ann Neurol 2004;55:736-9.

[11] Neychev VK, Gross R, Lehéricy S, Hess EJ, Jinnah HA. The functional neuroanatomy of dystonia. Neurobiol Dis 2011;42:185-201.

[12] De Vries PM, Johnson KA, de Jong BM, et al. Changed patterns of cerebral activation related to clinically normal hand movement in cervical dystonia. Clin Neurol Neurosurg 2008;110:120-8.

[13] De Vries PM, de Jong BM, Bohning DE, et al. Reduced parietal activation in cervical dystonia after parietal TMS interleaved with fMRI. Clin Neurol Neurosurg 2012;114:914-21.

[14] Koch G, Rothwell JC. TMS investigations into the task-dependent functional interplay between human posterior parietal and motor cortex. Behav Brain Res 2009;202:147-52.

[15] Civardi C, Cantello R, Asselman P, Rothwell JC. Transcranial magnetic stimulation can be used to test connections to primary motor areas from frontal and medial cortex in humans. Neuroimage 2001;14:1444-53.

[16] Koch G, Fernandez Del Olmo M, Cheeran B, et al. Focal stimulation of the posterior parietal cortex increases the excitability of the ipsilateral motor cortex. J Neurosci 2007;27:6815-22.

[17] Koch G, Fernandez Del Olmo M, Cheeran B, et al. Functional interplay between posterior parietal and ipsilateralmotor cortex revealed by twin-coil transcranial magnetic stimulation during reach planning toward contralateral space. J Neurosci 2008;28:5944-53.

[18] Koch G, Cercignani M, Pecchioli C, et al. In vivo definition of parieto-motor connections involved in planning of grasping movements. Neuroimage 2010; 51:300-12.

[19] Van Der Werf J, Jensen O, Fries P, Medendorp WP. Neuronal synchronization in human posterior parietal cortex during reach planning. J Neurosci 2010;30: $1402-12$.

[20] Vicario CM, Martino D, Koch G. Temporal accuracy and variability in the left and right posterior parietal cortex. Neuroscience 2013;245:121-8.

[21] Jahanshahi M, Rowe J, Fuller R. Impairment of movement initiation and execution but not preparation in idiopathic dystonia. Exp Brain Res 2001;140:460-8.

[22] Palomar FJ, Conde V, Carrillo F, et al. Parieto-motor functional connectivity is impaired in Parkinson's disease. Brain Stimul 2013;6:147-54.

[23] Herwing U, Satrapi P, Schönfeldt-Lecuona C. Using the international 10-20 EEG system for positioning of transcranial magnetic stimulation. Brain Topogr 2003;16:95-9.

[24] Rushworth MF, Taylor PC. TMS in the parietal cortex: updating representations for attention and action. Neuropsychologia 2006;44:2700-16.
[25] Koch G, Cercignani M, Bonnì S, et al. Asymmetry of parietal interhemispheric connections in humans. J Neurosci 2011;31:8967-75.

[26] Koch G, Bonnì S, Giacobbe V, et al. $\theta$-burst stimulation of the left hemisphere accelerates recovery of hemispatial neglect. Neurology 2012;78: 24-30.

[27] Caspers S, Eickhoff SB, Geyer S, et al. The human inferior parietal lobule in stereotaxic space. Brain Struct Funct 2008;212:481-95.

[28] Rossini PM, Barker AT, Berardelli A, et al. Non-invasive electrical and magnetic stimulation of the brain, spinal cord and roots: basic principles and procedures for routine clinical application. Report of an IFCN committee. Electroencephalogr Clin Neurophysiol 1994;91:79-92.

[29] Koch G, Oliveri M, Cheeran B, et al. Hyperexcitability of parietal-motor functional connections in the intact left-hemisphere of patients with neglect. Brain 2008; $131: 3147-55$

[30] Koch G, Ribolsi M, Mori F, et al. Connectivity between posterior parietal cortex and ipsilateral motor cortex is altered in schizophrenia. Biol Psychiatry 2008;64:815-9.

[31] Makris N, Kennedy DN, McInerney S, et al. Segmentation of subcomponents within the superior longitudinal fascicle in humans: a quantitative, in vivo, DT-MRI study. Cereb Cortex 2005;15:854-69.

[32] Delnooz CC, Helmich RC, Toni I, van de Warrenburg BP. Reduced parietal connectivity with a premotor writing area in writer's cramp. Mov Disord 2012;27:1425-31.

[33] Carboncini MC, Manzoni D, Strambi S, et al. Impaired agonists recruitment during voluntary arm movements in patients affected by spasmodic torticollis. Arch Ital Biol 2004;142:113-24.

[34] Boccagni C, Carpaneto J, Micera S, Bagnato S, Galardi G. Motion analysis in cervical dystonia. Neurol Sci 2008;29:375-81.

[35] Gregori B, Agostino R, Bologna M, et al. Fast voluntary neck movements in patients with cervical dystonia: a kinematic study before and after therapy with botulinum toxin type A. Clin Neurophysiol 2008;119:273-80.

[36] De Beyl DZ, Salvia P. Neck movement speed in cervical dystonia. Mov Disord 2009;26:2267-71.

[37] Anastasopoulos D, Ziavra N, Pearce R, Bronstein AM. Trunk bradykinesia and foveation delays during whole-body turns in spasmodic torticollis. J Neurol 2013;260:2057-65.

[38] Gilio F, Currà A, Inghilleri M, et al. Abnormalities of motor cortex excitability preceding movement in patients with dystonia. Brain 2003;126:1745-54.

[39] Davare M, Rothwell JC, Lemon RN. Causal connectivity between the human anterior intraparietal area and premotor cortex during grasp. Curr Biol 2010; 20:176-81.

[40] Talelli P, Ewas A, Waddingham W, et al. Neural correlates of age-related changes in cortical neurophysiology. Neuroimage 2008;40:1772-81.

[41] Prudente CN, Hess EJ, Jinnah HA. Dystonia as a network disorder: what is the role of the cerebellum? Neuroscience 2014;260:23-35. 